\title{
Hybrid RANS-LES vs URANS simulations of a simplified compressor blades cascade
}

\author{
Y. Hoarau, D. Szubert and M. Braza
}

\begin{abstract}
Based on a previous experience from BR710 and BR725 Roll-Royce fan blade development programs, the phenomena of shock wave-boundary layer interaction (SWBLI) are known to be of critical importance regarding engine safety and performance. In the european TFAST Project (Transition Location Effect on Shock Wave Boundary Layer Interaction), experiments and numerical simulations of unsteady and transitional SWBLI phenomena in a simplified compressor blades cascade are performed. Two blades of $100 \mathrm{~mm}$ are set-up in a $100 * 100 \mathrm{~mm}$ channel. The grid used is $9,6 \mathrm{M}$ cells with $y^{+}<1$ everywhere. The inlet turbulence intensity is set to $4 \%$ and the Mach number has been adjusted to be 1.22 in front of the lower blade. In the present paper, unsteady transitional RANS and hybrid RANSLES computations of the flow around two compressor blades are performed with the NSMB solver. Simulations with Spalart-Allmaras and $k-\omega$ turbulence models in their URANS and DDES formulation as well as $k-\omega$ SAS are performed.
\end{abstract}

\section{Introduction}

Vision-2020, whose objectives include the reduction of emissions and a more effective transport systems, puts severe demands on aircraft velocity and weight. These require an increased load on wings and aero-engine components. The greening of air transport systems means a reduction of drag and losses, which can be obtained by keeping laminar boundary layers on external and internal airplane parts. Increased

\footnotetext{
Y. Hoarau

ICUBE, CNRS - Université de Strasbourg - ENGEES - INSA, Strasbourg, France, e-mail: hoarau@unistra.fr

D. Szubert

IMFT, CNRS - INPT - Université de Toulouse, Toulouse, France, e-mail: dszubert@imft.fr

M. Braza

IMFT, CNRS - INPT - Université de Toulouse, Toulouse, France, e-mail: braza@imft.fr
} 
loads make supersonic flow velocities more prevalent and are inherently connected to the appearance of shock waves, which in turn may interact with a laminar boundary layer. Such an interaction can quickly cause flow separation, which is highly detrimental to aircraft performance, and poses a threat to safety. In order to diminish the shock induced separation, the boundary layer at the point of interaction should be turbulent, so that transition to turbulence does not occur within the SWBLI region. The main objective of this study, which is conducted within the European TFAST project, is to evaluate the effect of transition location on the SWBLI structure. The main question is how close the induced transition may be to the shock wave while still maintaining a typical turbulent character of interaction. The main study cases of this work deals with compressor blades and will help to answer open questions posed by the aeronautics industry and to tackle more complex applications. In the case of a civil turbofan engine operating at particularly high altitudes the Reynolds number can drop by a factor of 4 , when compared to the see level values. The laminar boundary layer on the transonic compressor rotor blades will interact with shock waves and as a result a strong boundary-layer separation will form. This can seriously affect the aero-engine performance and operation. One way to avoid strong separation is to ensure that the boundary layer upstream of the shock wave is turbulent. Forcing transition within the boundary layer can be achieved through the application of a surface roughness or a turbulator patch. Although such passive control methods are already in use, the mechanism of the shock wave/laminar boundary layer interaction, and in particular the source of the strong shock unsteadiness are still not well understood. Furthermore, the benefits of boundary layer control obtained for low Reynolds numbers can turn into loss increase at the higher levels of Reynolds numbers. Another possibility of transition control is to use Vortex Generators driven by Air Jet (AJVG). In the compressor application the jets may be driven by the pressure difference between the suction and pressure sides of the blade. There are two effects which should be investigated. The main effect is coming from streamwise vortices generated on the blade suction side but another is the suction taking place at the blade pressure side. This study takes place in the Work Package 3 of the TFAST project whose main goal is to improve the understanding of the shock wave/laminar boundary layer interaction on the transonic compressor blade. This can potentially lead to successful new design solutions.

Shock wave/turbulent boundary layer interactions (SWBLIs) are very complex flow phenomena which can be encountered in many flows such as transonic airfoils, supersonic inlets, aircraft wings, missiles, jets, nozzles... Most of the time the shock interacts significant with the incoming boundary layer leading to separation which can give rise to unsteady flow (buffeting, inlet instability, ...). SWBLI has been extensively studied over the past 50 years. Review of such work can be found in $[1,2,3,5,6,7,10,14] \ldots$

The unsteadiness generated by SWBLI is characterized by a wide range of frequencies, which encompass the characteristics of the upstream boundary layer as well as motions that are typically one to two orders of magnitude lower.

The physical source of this unsteadiness is still unclear and in a recent review, [4] concluded that both fluctuations in the upstream boundary layer and large-scale in- 
stability intrinsic to the separated flow but the downstream mechanism dominates for strongly separated flows, and a combined mechanism dominates for weakly separated flows.

In this paper we compare the result of the modeling of SWBLI in a simplified compressor cascade with RANS and DDES/SAS models. This work is preliminary in the sense that no transition modeling has been introduced and the flow is fully turbulent. The next section presents details of the test configuration and of the numerical aspects of the simulations. Results and discussion are developed in section 3.

\section{Case Description and Numerical modeling}

The selected geometry is representative of a Low Pressure Compressor first rotor at mid-span. The Mach number in front of the blade is 1.2 , the deflection $15^{\circ}$ and the Axial Velocity Density Ratio 1.2. The original geometry has been scaled to a chord of $100 \mathrm{~mm}$ to fit in the transonic wind tunnel of IMP-PAN, Gdansk, Poland (figure 1). Fiew results of these experiments are available when this paper has been writen. Between the sea level and the cruise altitude of $51 \mathrm{kft}$, the Reynolds Number drops by a factor of 4 . Preliminary simulations with $\mathrm{Tu}=0.5 \%$ and $4.0 \%$ show that at low Tu and altitude of $45 \mathrm{kft}$ (typical cruise), the suction surface boundary layer upstream of shock is fully laminar which is the main requirement for the design of the test section. The geometry simulated in this paper is presented in the figure 2 and is around $2 \mathrm{~m}$ length and $100 \mathrm{~mm}$ in the spanwise direction. The inlet Mach number is 0.5 and the outlet pressure has been calibrated to have a Mach number of 1.22 in front of the first blade. The grid was generated by NUMECA/Autogrid and the $y^{+}$is lower than 1 in all the regions of interest. The size of the 3D grid is $9.6 \times 10^{6}$ cells. The highest Reynolds number corresponding to the sea level altitude, $R e=4.4 \times 10^{6}$, and an inlet turbulence intensity of $4 \%$ are used.

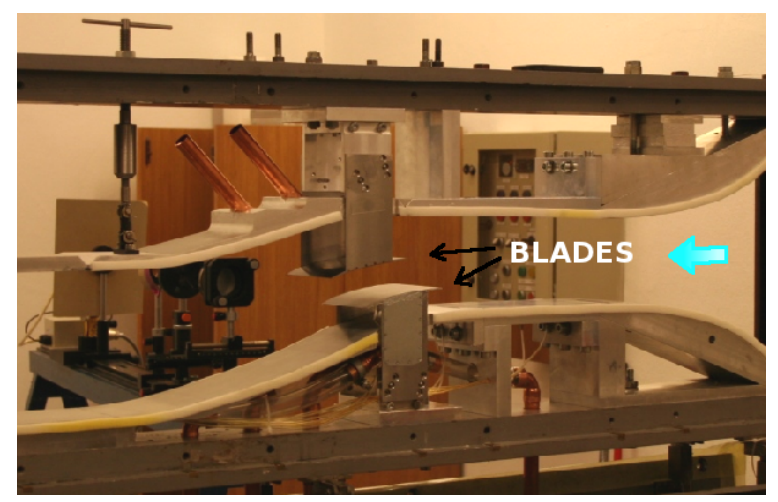

Fig. 1 Experimental setup (IMP-PAN, Gdansk, Pologne) 


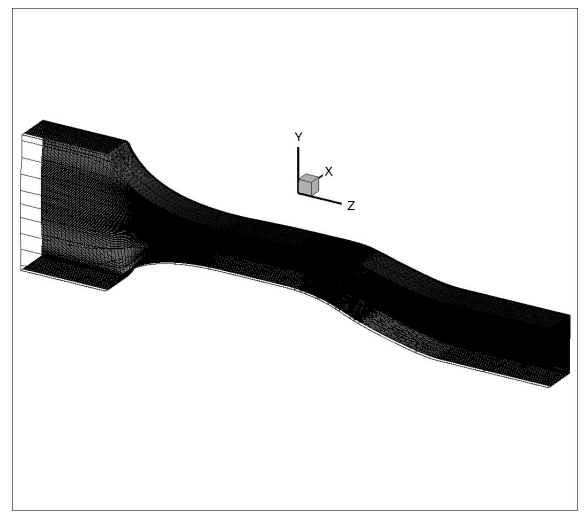

a)

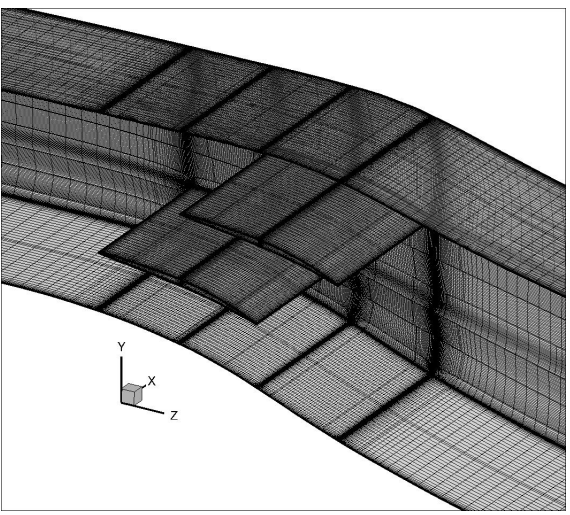

b)

Fig. 2 a) View of the whole grid and b) details of the compressor cascade

In this study the compressible NSMB flow solver [17] has been used. The NSMB code solves the compressible Navier-Stokes equations using a finite volume formulation on structured Multi-Block grids and is parallelized using the Message Passing Interface. Among the many discretization schemes available in NSMB, in this study we use the $3^{\text {rd }}$ order AUSMPW+ upwind scheme [9] for the convection and a second order central scheme for the diffusion. An implicit dual-time stepping solved by Lower-Upper Symmetric Gauss-Seidel method (LS-SGS) is used for the time integration. The time-step is $\Delta t=10^{-5} s$ and the inner loop is stopped when the convergence reaches $10^{-3}$ for each time-step which correspond approximatively to 60-70 iterations. Two URANS turbulence models have been used, the [8] version of the one-equation model of [15] (SA) and the two-equation $k-\omega$ SST model of [11] as well as three hybrid RANS/LES versions of these models, the Delayed Detached Eddy Simulation ([16]) of the SA and the $k-\omega$ SST models as well as the $k-\omega$ SST Scale Adaptive Simulation model of $[12,13]$. As previously mentioned the flow simulated here is fully turbulent.

\section{Results and discussion}

A Schlieren visualization of the experimental flow is provided in figure 3 (a), as well as a distribution of the Mach number over the upper side of the lower blade (b), carried out by IMP-PAN, Gdansk, in the framework of the TFAST European programme. A reduction of the Mach number is observed across the shock generated by the upper blade and impinging the lower one. The evolution of the experimental isentropic Mach number for both fully turbulent and laminar case are presented for the extrados on the lower blade and the results are in fair agreement with the numerical SA-DDES. In simulations, the flow is characterised by the formation of 
five shocks : the first one forms in front of the lower blade, the second between the lower wall and the first blade, the third on the suction side of the first blade and in front of the second blade, the fourth one between the two blades and the last one between the second blade and the upper wall. In all the simulations the mach number before the first shock is around $M=1.22$. The overall flow structure shown in figure 4 displays this shock-waves formation in good qualitative comparison with the Schlieren visualization ( 3 a), in particular the SA model that gave a good aspect of the first and third shocks, on front of each blade. Shocks between the upper and lower walls and the blade are not visible due to the experimental setup. This first shock is detached from the lower blade for the RANS models and oscillates on a small distance at a frequency around $160-173 \mathrm{~Hz}$, whereas for the DDES models the shock moves on a bigger distance and come very closed to the lower blade with a frequency around $114-121 \mathrm{~Hz}$. The frequency is lower because the extent of the movement is much higher. In the case of the SAS model, this shock is very oblique, almost attached to the lower blade and mainly it is steady as well as the third shock which is also oblique, very closed to the second blade and steady whereas for the RANS and the DDES models it oscillates around the same frequency $(210-235 \mathrm{~Hz})$. The second shock below the first blade oscillates on a very large distance, $\approx 25 \%$ of the chord, at a frequency of $307-364 \mathrm{~Hz}$ for both RANS and DDES models. In the DDES simulations the fourth shock between the two blades remains closed to the trailing edge and moves on a short distance in a very erratic way so that it is very difficult to extract a frequency. In the RANS simulations the shock moves on $\approx 20 \%$ of the chord up to the trailing edge but its behavior is also very erratic. Finally the opposite behavior is observed of the last shock between the upper wall and the second blade : it's amplitude is higher in the DDES simulation than with the RANS models.
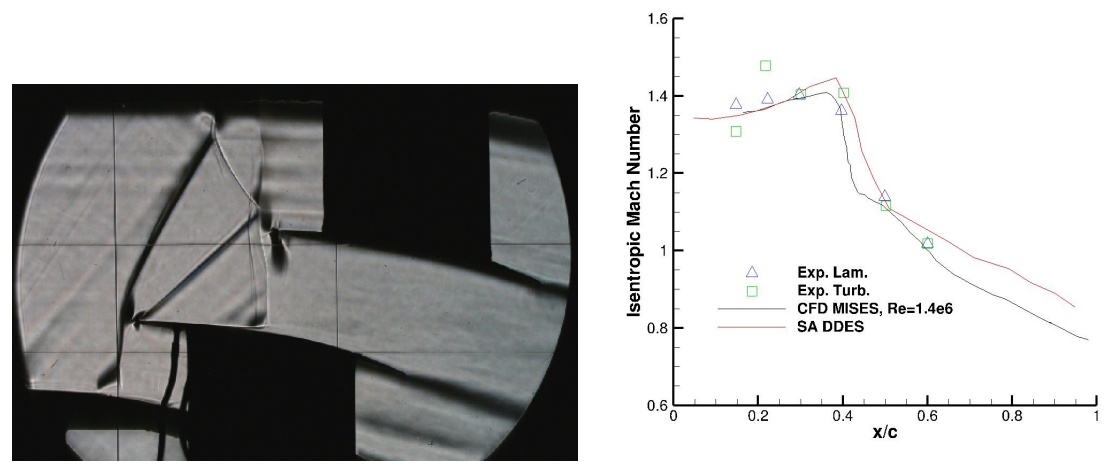

a)

Fig. 3 Schlieren visualisation of the experimental flow a) and comparison of the Isentropic-Mach number distribution over the upper side of the lower blade b) (IMP-PAN, Gdansk, Pologne) 

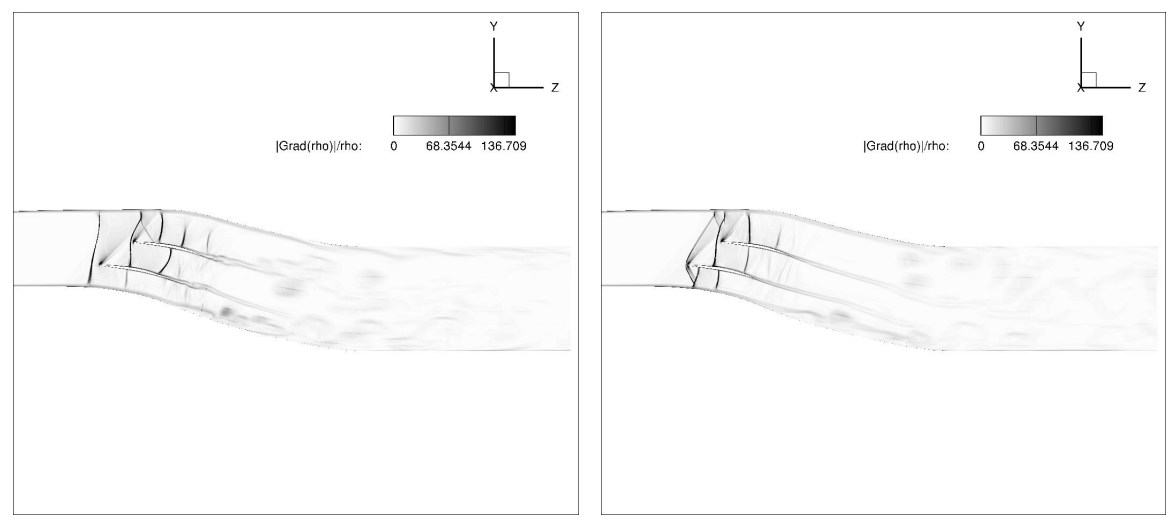

a)
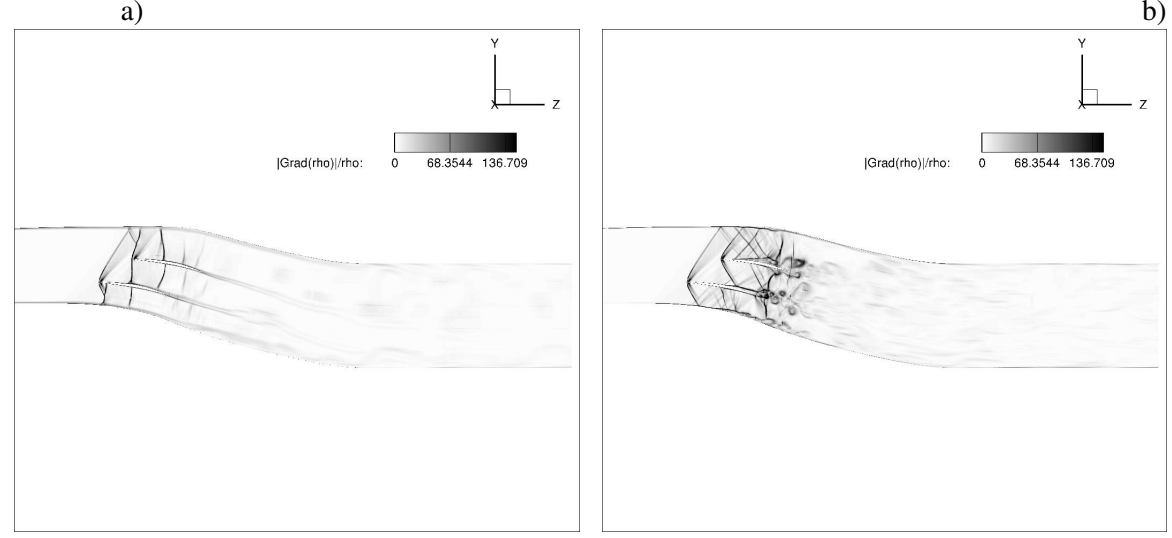

c)

d)

Fig. 4 Schlieren visualisation of the flow in the middle plane : a) SA model, b) SA DDES, c) $k-\omega$ SST DDES and d) $k-\omega$ SST SAS

Figure 5 features the time evolution of the lift coefficient on each blade. On the lower blade all the models except for the $k-\omega$ SST SAS give almost the same evolution, the SAS having a lower mean value whereas on the second blade all the five simulations stand in the same range. This is confirmed in the table 1, where the mean values of the aerodynamic coefficients for the five models are presented. The SAS model gives a much lower value of the drag and lift coefficients on the lower blade and on the second blade the drag of the SAS is slightly higher. All the other models show comparable values. From the lift coefficient time evolution on the second blade of the $k-\omega$ SST model, we can extract a low frequency of $387 \mathrm{~Hz}$ for the SWBLI. This frequency in fact exists for all the models on each blade but there are a lot of sub-harmonics for the other models. Although the aerodynamic coefficients are quite comparable, the main flow feature is different between RANS and hybrid models, as can be seen on the figure 4. 


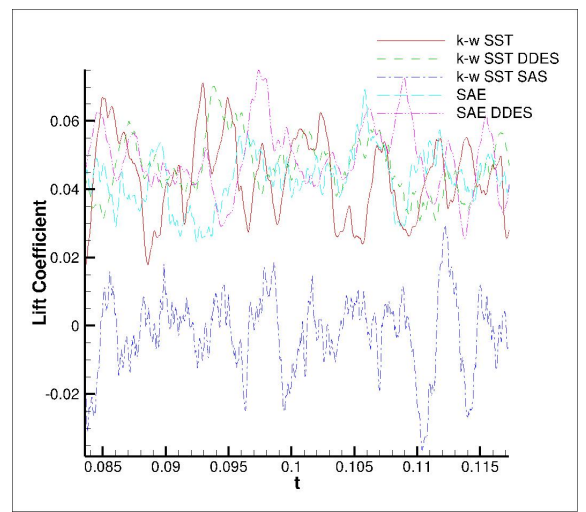

a)

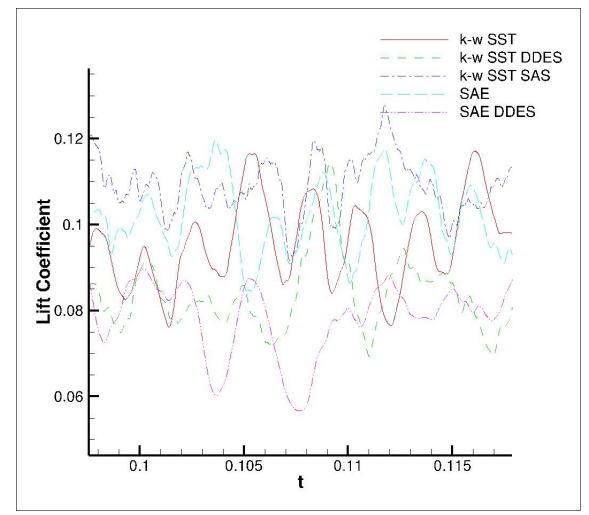

b)

Fig. 5 Time evolution of the lift coefficient on the lower blade a) and the upper blade b) for all the turbulence models.

Table 1 Mean values of drag and lift coefficients on each blade

\begin{tabular}{clcc}
\hline & & $C_{D}$ & $C_{L}$ \\
\hline \multirow{5}{*}{$1^{\text {st }}$ blade } & SAE & 0.0079 & 0.0412 \\
& SAE DDES & 0.0095 & 0.0506 \\
& $k-\omega$ SST & 0.0078 & 0.0377 \\
& $k-\omega$ SST DDES & 0.0080 & 0.0437 \\
& $k-\omega$ SST SAS & 0.00074 & 0.0082 \\
\hline \multirow{4}{*}{$2^{\text {nd }}$ blade } & SAE & 0.0209 & 0.1045 \\
& SAE DDES & 0.0171 & 0.0859 \\
& $k-\omega$ SST & 0.0195 & 0.0982 \\
& $k-\omega$ SST DDES & 0.0183 & 0.0917 \\
& $k-\omega$ SST SAS & 0.0257 & 0.1053 \\
\hline
\end{tabular}

The SWBLI is much more important in the case of the SAS model mainly because with this model the flow is laminar around the airfoils and on the upper and lower walls or at least the turbulence intensity is extremely small compared to the RANS ans the DDES simulations, as can be seen on the figure 6 . The boundary layers are very sensitive to the pressure variation generated by the shocks and we can observe massive separation on each blade as well as on the upper and the lower wall. The side walls also introduce corner vortices which are captured by all the turbulence models but the SAS model gives much smaller structures than the other models. 

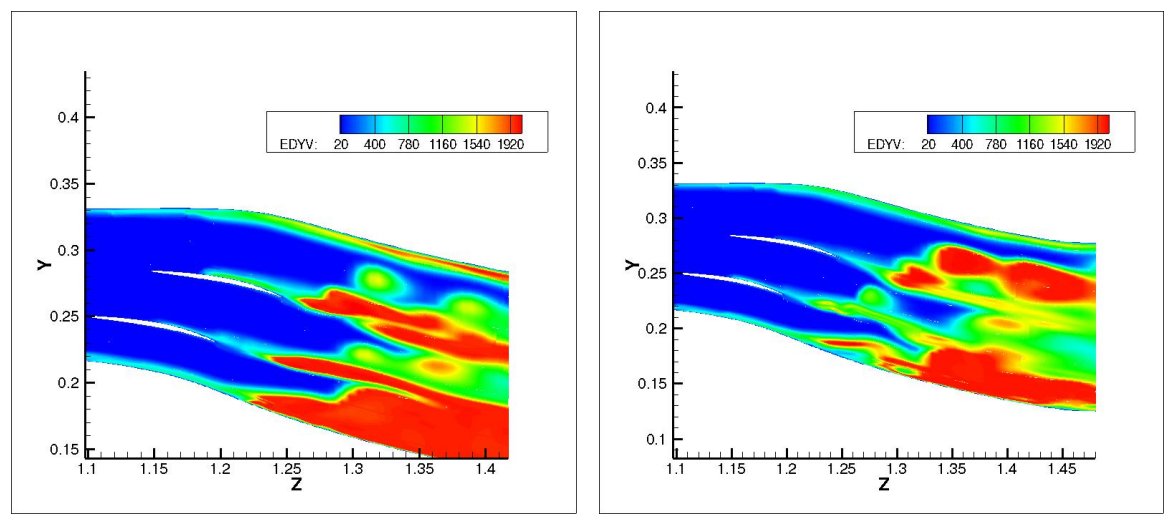

a)
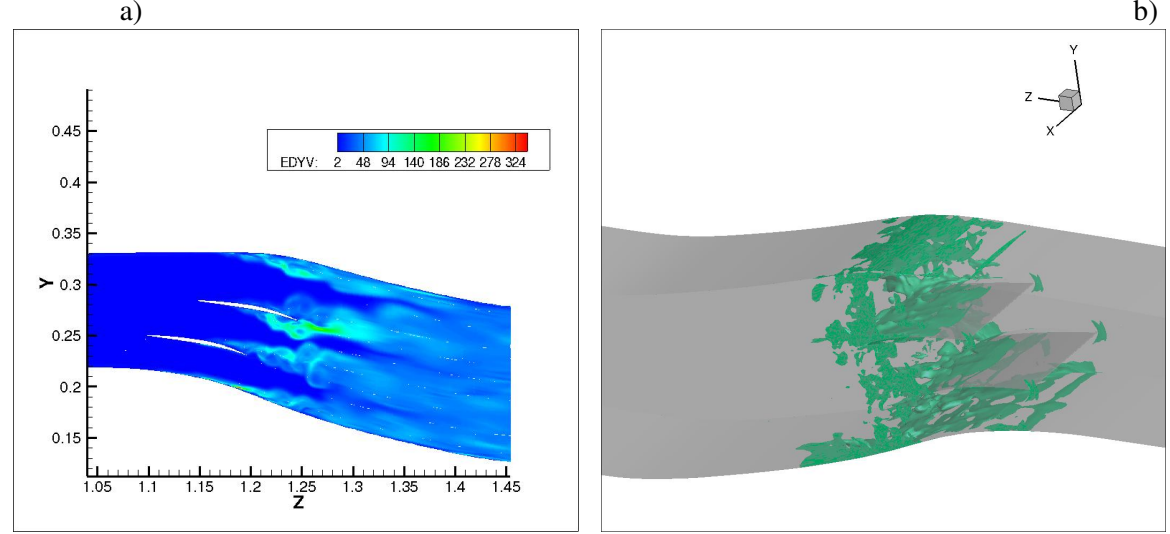

c)

Fig. 6 Turbulent viscosity in the middle plane for the $k-\omega$ SST a), $k-\omega$ SST DDES b), $k-\omega$ SAS c) and iso-suface of negative streamwise velocity $(W=-1 \mathrm{~m} / \mathrm{s})$ for the $k-\omega$ SAS model.

\section{Conclusion}

The flow around a simplified configuration of a low pressure compressor has been simulated in the preliminary configuration of the wind tunnel of IMP-PAN, Gdansk. The Reynolds number of $R e=4.4 \times 10^{6}$ corresponds to flight at sea level altitude. The flow is fully turbulent with an inlet intensity of $4 \%$. Five simulations have been completed : two RANS and three hybrid RANS/LES. The RANS and the DDES present globally the same flow features qualitatively comparable with the experiment, the differences on the shocks location and oscillations having no tremendous effects on the mean aerodynamics coefficients whereas with the SAS model, a very small turbulence level is predicted which give rise to massive separation induced by the shock wave boundary layer interaction. 
Acknowledgements This work has been carried out in the context of the European project TFAST. We acknowledge the CPU allocation on the French Supercomputing Centres IDRIS and CINES.

\section{References}

1. Babinsky, H., Harvey, J. K., 2011. Shock WaveBoundary-Layer Interactions. Cambridge Univ. Press.

2. Bouhadji, A., Braza, M., 2003. Physical analysis by numerical simulation of organised modes and shock-vortex interaction in transonic flows around an aerofoil. Part II: Reynolds number effect., J. Computers and Fluids, Vol. 32(9), pp. 1261-1281.

3. Bourdet, S., Braza, M., Bouhadji, A., Thiele, F., 2003. Direct Numerical Simulation of the three-dimensional transition to turbulence in the transonic flow around a wing. J. Flow Turbulence and Combustion, Vol. 71, pp 203-220.

4. Clemens, N. T., Narayanaswamy, V., 2014. Low-Frequency Unsteadiness of Shock Wave/Turbulent Boundary Layer Interactions. Annu. Rev. Fluid Mech. Vol. 46, pp 469-492

5. Doerffer, P., Hirsch C., Dussauge, J. P., Babinsky, H., Barakos, G. N., 2010. Unsteady Effects of Shock Wave Induced Separation. New York: Springer

6. Dolling, D. S., 2001. Fluctuating loads in shock-wave /boundary layer interaction: tutorial and update. AIAA Pap. 1993-0284.

7. Dolling, D. S., 2001. Fifty years of shock wave/boundary layer interaction: what next? AIAA J., Vol. 39, pp 1517-1531.

8. Edwards, J. R., Chandra, S., 1996. Comparison of Eddy Viscosity-Transport Turbulence Models for Three-Dimensional, Shock-Separated Flowfields. AIAA Journal, Vol. 34(4), pp 756763.

9. Kim, K. H., Kim, C., Rho, O.-H., 2001. Methods for the Accurate Computations of Hypersonic Flows: I. AUSMPW+Scheme. J. Of Computational Physics, Vol. 174 (1), pp 38-80.

10. Lee, B. H. K., 2001. Self-sustained oscillations on airfoils at transonic speeds. Prog. Aerosp. Sci., Vol. 37, pp 147-96.

11. Menter, F., 1994. Two-equation eddy-viscosity turbulence models for engineering applications. AIAA J. 32(8), 1598-1605.

12. Menter, F., Bender, R., 2003. A scale-adaptive simulation model for turbulent flow prediction. AIAA paper 2003-0767, Reno, NV.

13. Menter, F., Egorov, Y., 2005. A scale-adaptive simulation model using two-equation models. AIAA paper 2005-1095, Reno, NV.

14. Smits, A. J., Dussauge, J. P., 1996. Turbulent Shear Layers in Supersonic Flow. Woodbury, NY: AIP.

15. Spalart, P., Allmaras, S., 1992. A one-equation turbulence model for aerodynamic flows. AIAA Paper 439.

16. Spalart, P., Deck, S., Shur, M., Squires, K., Strelets, M., Travin, A., 2006. A new version of detached-eddy simulation, resistant to ambiguous grid densities. Theor. Comput. Fluid Dyn. 20, 181-195.

17. Vos, J., Chaput, E., Arlinger, B., Rizzi, A., Corjon, A., 1998. Recent advances in aerodynamics inside the NSMB (Navier-Stokes Multi-Block) consortium. AIAA Paper 0802. 